\title{
Top-down Tree Transducers with Regular Look-ahead
}

\author{
by \\ JOOST ENGELFRIET* \\ Twente University of Technology \\ Enschede, Netherlands \\ ABSTRACT
}

Top-down tree transducers with regular look-ahead are introduced. It is shown how these can be decomposed and composed, and how this leads to closure properties of surface sets and tree transformation languages. Particular attention is paid to deterministic tree transducers.

Introduction. The top-down finite state tree transformations discussed in, for example, $[3,7,13,14]$ fail to have certain nice closure properties with respect to composition. It was argued in [7] that this is due to the fact that a top-down tree transducer cannot inspect a subtree before deleting it (a property possessed by bottom-up tree transducers). In this paper we add the facility of regular lookahead to the usual type of top-down tree transducer. The transducer is now allowed to inspect the subtrees of a node before processing it (thus having an arbitrarily large look-ahead). However, the look-ahead is restricted in that the information which the transducer extracts from the șubtrees should be finite and even regular (or "recognizable" i.e. computable by a finite tree automaton). We note that the idea of regular look-ahead also occurs in the theory of parsing of context-free languages [4]. It turns out that the class of tree transformations realized by this type of transducer has all the expected closure properties with respect to composition, for instance, both the classes of linear and deterministic top-down tree transformations are now closed under composition (we note here that composition results can also be proved for restricted types of top-down tree transducers, such as total or nondeleting ones, see $[13,14]$ ). These composition results are proved, as in [7], by first decomposing the transformations into simpler parts and then showing composition properties of these simpler transformations. In fact, any top-down tree transducer with regular look-ahead can be realized in two phases. The first phase (which can be accomplished bottom-up and deterministically) computes all the look-ahead information and stores it in the labels at the nodes of the input tree. The second phase is an ordinary top-down tree transducer which uses this information to imitate the one with regular look-ahead. This decomposition result is also useful in obtaining results about top-down finite state tree transformations without regular lookahead, in particular concerning their surface sets.

*The research reported here was carried out during a one-year visit of the author to the Dept. of Computer Science of Aarhus University, Aarhus, Denmark. 
This paper is a sequel to [7] and the reader is assumed to be familiar with the methods and results of [7]. However, we do not assume any familiarity with section 5 of [7]. We note that the class $T^{\prime}-F S T$, defined in that section, is in fact equal to the class of top-down tree transformations with regular look-ahead (cf. the remarks following [7, Theorem 5.13]).

In section 1 we list some changes in terminology with respect to [7], some additional terminology, and some additional lemmas.

In section 2 we define the top-down tree transducer with regular look-ahead and show the above mentioned decomposition and composition results.

In section 3 we compare the deterministic bottom-up and top-down tree transformations. The deterministic bottom-up tree transformations are (properly) contained in the deterministic top-down tree transformations with regular look-ahead.

In section 4 we apply the results of the previous sections to top-down surface sets and yields of surface sets. It follows for instance from the result in section 3 that the deterministic top-down surface sets are closed under deterministic bottom-up tree transformations. We finally mention possible applications to Lindenmayer languages.

\section{Preliminaries}

The reader is referred to [7] for all unexplained terminology. That paper will from now on be referred to as $[B T]$ rather than [7].

We recall that we often make no explicit distinction between a transducer $\langle\Sigma$, $\Delta, Q, Q_{d}, R>$ and the transformation from $T_{\Sigma}$ to $T_{\Delta}$ that it computes. We also recall that by the relational composition $R_{1} \circ R_{2}$ we mean "first $R_{1}$, then $R_{2}$ "'. We finally restate the important properties $(B 1),(B 2)$ and $(T)$ of $[B T]$.

(B1) Copying of an output tree after nondeterministic processing of the input tree.

(B2) Deciding whether to delete a tree or not after processing it.

(T) Copying of an input tree and processing the copies differently.

In the rest of this section we list some changes in and additions to the terminology in $[B T]$. Some additional facts, to be used in later sections, are also mentioned.

First we change our use of "deterministic top-down" and of "DT" so as to agree with [13]. A top-down $f_{s t}\left\langle\Sigma, \Delta, Q, Q_{d}, R\right\rangle$ will be called deterministic if (1) $Q_{d}$ is a singleton and (2) different rules in $R$ have different lefthand sides. It is easy to see that every deterministic top-down $f_{s t}$ is equivalent to one which processes the whole input tree (except eventually for its leaves) and then decides whether to accept it or not. The version in $[B T]$ will be called a total deterministic $t$-fst (since it accepts every input tree). Determinism will be denoted as usual by a $D$, so that $D T-F S T$ denotes the class of deterministic top-down $f_{s t}$ (and not the class of total deterministic top-down $f s t$, as was the case in $[B T]$ ). The class of linear deterministic top-down fst will be denoted by LDT-FST. The definition of HOM and $L H O M$ is not changed, i.e. homomorphisms are total.

Secondly, we shall write $R E L$ rather than $R E L A B$. Thus $Q R E L$ denotes the class of (bottom-up or top-down) finite state relabelings (cf. Definition 3.14 of 
$[B T]$ ). The class of deterministic bottom-up finite state relabelings will be denoted by $D B Q R E L$ (this class was denoted DQRELAB in [BT]), and the class of deterministic top-down finite state relabelings by DTQREL.

We shall use the following additional decomposition results, the detailed proof of which is left to the reader.

\section{LEMMA 1.1.}

$$
\begin{gathered}
T-F S T \subseteq H O M \circ L T F S T \\
D T-F S T \subseteq H O M \circ L D T-F S T, \\
L D T-F S T \subseteq D T Q R E L \circ L H O M .
\end{gathered}
$$

Proof. (1) The first inclusion is shown in [BT, Lemma 3.6] and the second inclusion easily follows from the proof of that lemma.

(2) The proof of this inclusion is similar to that of [BT, Theorems 3.5 and 3.15]. Roughly, for $T$ in $L D T-F S T$, one can construct $T_{1}$ in DTQREL and $T_{2}$ in LHOM such that the $i$-th rule $q\left(\sigma\left(x_{1} \ldots x_{k}\right)\right) \rightarrow t$ of $T$ is split into two rules $q\left(\sigma\left(x_{1} \ldots x_{k}\right)\right) \rightarrow i\left(q_{1}\left(x_{1}\right) \ldots q_{k}\left(x_{k}\right)\right)$ of $T_{1}$ and $*\left(i\left(x_{1} \ldots x_{k}\right)\right) \rightarrow t\left[*\left(x_{1}\right), \ldots\right.$, $\left.*\left(x_{k}\right)\right]$ of $T_{2}$, where $*$ is the only state of $T_{2}$, and where, for each $j(1 \leq j \leq k)$, either $q_{j}\left(x_{j}\right)$ occurs in $t$ or $q_{j}=q_{0}$ (and $q_{0}$ is a new state which is, for instance, the identity on all trees).

We shall also use the following result (cf. [12]).

LEMMA 1.2. Let $F$ be the composition of a finite number of bottom-up fst, i.e. $F=B_{1} \circ B_{2} \circ \ldots \circ B_{n}$ for some $n \geq 1$ and $B_{i} \in B-F S T$. Then

(1) $R E C O G$ is closed under $F^{-1}$ (i.e. if $L \in R E C O G$, then $F^{-1}(L) \in R E C O G$ ), and

(2) $\operatorname{dom}(F) \in R E C O G$.

Proof. (1). It obviously suffices to assume that $F \in B-F S T$. Let $L$ be a recognizable tree language and let $R$ be a finite tree automaton with domain $L$. Then $F^{-1}(L)=\operatorname{dom}(F \circ R)$. By $[B T$, Lemma 4.2(1)] $F \circ R \in B-F S T$ and hence its domain is recognizable by [BT, Corollary 3.12]. Statement (2) is immediate from (1) by the fact that $\operatorname{dom}(F)=F^{-1}\left(T_{\Delta}\right)$, where $\Delta$ is the output alphabet of $F$. $\square$

Note that, since FTA, REL and HOM are included in B-FST, the decomposition result for top-down $f s t[B T$, Theorem 3.9] implies that Lemma 1.2 also holds with "bottom-up" replaced by "top-down" ([12]).

We finally introduce some more terminology concerning surface sets and tree transformation languages.

Let $\mathscr{L}$ be a class of tree languages and $\mathscr{F}$ a class of tree transformations. Then $\mathscr{F}(\mathscr{L})$ denotes the class of tree languages $\{F(L) \mid F \in \mathscr{F}$ and $L \in \mathscr{L}\}$, which we shall call $(\mathscr{F} . \mathscr{L})$ surface sets. If $\mathscr{L}=$ RECOG then the $(\mathscr{F}, \mathscr{L})$ surface sets are the $\mathscr{F}$ surface sets.

Let $e$ be a fixed symbol of rank 0 (which may or may not be an element of a ranked alphabet). The yield of a tree $t$, denoted by yield $(t)$, is the string defined recursively as follows: 
(1) for $\sigma$ of $\operatorname{rank} 0, \operatorname{yield}(\sigma)=\left\{\begin{array}{ll}\sigma & \text { if } \sigma \neq e \\ \lambda & \text { if } \sigma=e\end{array}\right.$ where $\lambda$ is the empty string;

(2) for $\sigma$ of rank $k \geq 1$ and trees $t_{1}, \ldots, t_{k}$,

$$
\operatorname{yield}\left(\sigma\left(t_{1} \ldots t_{k}\right)\right)=\operatorname{yield}\left(t_{1}\right) \ldots \operatorname{yield}\left(t_{k}\right) \text {. }
$$

Furthermore we define, for a tree language $L$, yield $(L)=\{\operatorname{yield}(t) \mid t \in L\}$ and, for a family $\mathscr{L}$ of tree languages, yield $(\mathscr{L})=\{\operatorname{yield}(L) \mid L \in \mathscr{L}\}$. Thus yield $(L)$ is a string language and yield $(\mathscr{L})$ is a family of string languages.

For a class $\mathscr{L}$ of tree languages and a class $\mathscr{F}$ of tree transformations, the class of languages yield $(\mathscr{F}(\mathscr{L})$ ) will be called the class of $(\mathscr{F}, \mathscr{L})$ tree transformation languages. Thus a tree transformation language is the yield of a surface set.

In the next lemma we show that in many cases we can do without the special symbol $e$ to denote $\lambda$. This lemma is in fact a particular case of Theorem 3.2.10 in [3].

LEMMA 1.3. Let $\mathscr{L}$ be a family of tree languages and $\mathscr{F}$ a family of tree transformations such that $\mathscr{F}(\mathscr{L})$ is closed under linear deterministic bottom-up fst. Let $L$ be an $(\mathscr{F}, \mathscr{L})$ tree transformation language i.e. $L \in$ yield $(\mathscr{F}(\mathscr{L})))$. Then $L-\{\lambda\}=$ yield $(F(M))$ for some $M \in \mathscr{L}$ and some $F \in \mathscr{F}$, such that the output alphabet of $\mathscr{F}$ does not contain $e$.

Proof. Let $L=\operatorname{yield}\left(G\left(M_{1}\right)\right)$ for some $M_{1} \in \mathscr{L}$ and $G \in \mathscr{F}$, and let $G\left(M_{1}\right)$ be over the ranked alphabet $\Sigma$ with $e \in \Sigma$ (otherwise there is nothing to prove). We now construct a linear deterministic bottom-up $f_{s t} B$ which, for any tree $t$ in $T_{\Sigma}$, deletes all subtrees $t_{1}$ of $t$ with yield $\left(t_{1}\right)=\lambda$ (and does not accept $t$ if yield $(t)=i$ ). In fact, $B=\left\langle\Sigma, \Delta, Q, Q_{\mathrm{d}}, R\right\rangle$ where $Q=\left\{q_{e}, q_{f}\right\}, Q_{d}=\left\{q_{f}\right\}, \Delta$ is the ranked alphabet such that, for each $k, \Delta_{k}=\Sigma-\{e\}$, and $R$ contains the following rules. First of all it contains rules $\sigma \rightarrow q_{f}(\sigma)$ for each $\sigma \in \Sigma_{0}-\{e\}$ and one rule $e \rightarrow q_{e}\left(\sigma_{0}\right)$ where $\sigma_{0}$ is an arbitrary element of $\Sigma_{0}-\{e\}$ (note that for $\Sigma_{0}=\{e\}$ the proof is trivial). Furthermore, for each $k \geq 1, \sigma \in \Sigma_{k}$ and $q_{1}, \ldots, q_{k} \in Q, R$ contains the rule $\sigma\left(q_{1}\left(x_{1}\right) \ldots q_{k}\left(x_{k}\right)\right) \rightarrow q\left(\sigma\left(x_{i_{1}} \ldots x_{i_{n}}\right)\right)$ where $q_{i_{1}}=q_{i_{2}}=\ldots=q_{i_{n}}=q_{f}$ $\left(1 \leq i_{1}<i_{2}<\ldots<i_{n} \leq k\right)$ and all other $q_{j}$ are equal to $q_{e}$, and $q=q_{e}$ if and only if $n=0$ (i.e. $q_{1}=q_{2}=\ldots=q_{k}=q_{e}$; in this case the righthand side of the rule is $\left.q_{e}(\sigma)\right)$. It is easy to prove that, if $t_{1} \stackrel{*}{\Rightarrow} q_{e}\left(t_{2}\right)$, then yield $\left(t_{1}\right)=\lambda$, and if $t_{1} \stackrel{*}{\Rightarrow} q_{j}\left(t_{2}\right)$, then yield $\left(t_{2}\right)=\operatorname{yield}\left(t_{1}\right) \neq \lambda$. Thus $L-\{\lambda\}=\operatorname{yield}\left(B\left(G\left(M_{1}\right)\right)\right)$ and, since $\mathscr{F}(\mathscr{L})$ is closed under $B, L-\{\lambda\}=\operatorname{yield}(F(M))$ for some $F(M) \in \mathscr{F}(\mathscr{L})$, where $F$ has output alphabet $\Delta$.

Note that, when a tree transducer, together with an input tree language, is viewed as a generating device of a tree transformation language, then Lemma 1.3 tells us that we can get rid of $\lambda$-rules.

\section{Top-down tree transducers with regular look-ahead; decomposition and composition}

In this section we add the facility of regular look-ahead to the top-down $f$ st. Consequently the top-down $f s t$ will be able to inspect a subtree in order to decide whether to delete it or not (cf. property (B2)). Thus the difference between bottomup fst and top-down $f$ st with regular look-ahead can then be characterized by properties $(B 1)$ and $(T)$. 
In order to define the top-down $f$ st with regular look-ahead we have to slightly generalize the notion of a semi-thue system with variables [BT, section 1]. We shall allow the range of the variables to be different for different rules. Formally we redefine a semi-thue system with variables to be a system $G=\langle A, X, R\rangle$, where $A$ is an alphabet, $X=\left\{x_{1}, x_{2}, \ldots\right\}$ and $R$ a finite set of rules of the form $\langle\phi \rightarrow \psi$, $D>$ such that, for some $k \geq 0, \phi$ and $\psi$ are in $\left(A \cup X_{k}\right)^{*}$ and $D$ is a mapping from $X_{k}$ into the powerset of $A^{*}$. For $1 \leq i \leq k, D\left(x_{i}\right)$ is called the range of $x_{i} ; \phi$ is called the lefthand side and $\psi$ the righthand side of the rule. Whenever $D$ is understood (in particular when $k=0, D$ is always empty) or will be specified later, we shall write $\phi \rightarrow \psi$ rather than $\langle\phi \rightarrow \psi, D\rangle$. The relations $\overrightarrow{\vec{G}}$ and $\underset{\vec{G}}{*}$ are defined as in $[B T]$, the only difference being that the mapping $D$ now depends upon the rule.

We now define a top-down $f_{\text {st }}$ with regular look-ahead to be a top-down $f_{s t}$ in which the ranges of the variables in each rule are certain recognizable tree languages.

Definition 2.1. A top-down finite state tree transformation with regular lookahead (abbreviated by $t^{r}$-fst) is a 5-tuple $T=\left\langle\Sigma, \Delta, Q, Q_{d}, R\right\rangle$, where $\Sigma, \Delta, Q$ and $Q_{d}$ are as for a top-down $f s t$ and $R$ is a finite set of rules of the form $\left\langle t_{1} \rightarrow t_{2}, D\right\rangle$, where $t_{1} \rightarrow t_{2}$ is an ordinary top-down $f$ st rule and $D$ is a mapping from $X_{k}$ into the powerset of $T_{\Sigma}$ (where $X_{k}$ is the set of variables occurring in $t_{1}$ ), such that, for $1 \leq i \leq k, D\left(x_{i}\right) \in R E C O G . T$ is viewed as the semi-thue system with variables $\langle\Sigma \cup \Delta \cup Q \cup\{()\}, X, R$,$\rangle , and the tree transformation defined by T$ is as usual $\left\{\langle t, s\rangle \in T_{\Sigma} \times T_{\Delta} \mid q(t) \stackrel{*}{\Rightarrow} s\right.$ for some $q$ in $\left.Q_{d}\right\}$.

The class of all $t^{r}$-fst will be denoted by $T^{R}-F S T$.

We note that it will always be assumed in a $t^{r}-f_{s t}$ that the ranges of the variables are specified in some effective way, for instance as deterministic bottomup finite tree automata. Throughout the paper all constructions will be effective in this sense.

Example 2.2. There is a $t^{r}$-fst that is not a $t$-fst. In fact, consider the bottom-up fst $B=\left\langle\Sigma, \Delta, Q, Q_{d}, R\right\rangle$ of $[B T$, Example 2.6] which is not a $t-f s t$. Let $U$ be the recognizable tree language $T_{\Omega}$, where $\Omega_{0}=\{b\}, \Omega_{1}=\{a\}$ and $\Omega_{2}=\{\sigma\}$. Consider now the $t^{\prime}$-fst $T=\left\langle\Sigma, \Delta, Q^{\prime}, Q_{d}^{\prime}, R^{\prime}\right\rangle$ where $Q^{\prime}=Q_{d}^{\prime}=\{*\}$ and $R^{\prime}$ consists of the rules

$$
\begin{aligned}
& \left\langle *(\sigma(x y)) \rightarrow \sigma(*(x)), D_{1}\right\rangle \text { with } D_{1}(x)=D_{1}(y)=U, \\
& \left\langle *(a(x)) \rightarrow a(*(x)), D_{2}\right\rangle \text { with } D_{2}(x)=T_{\Sigma}, \text { and } \\
& *(b) \rightarrow b .
\end{aligned}
$$

Then, obviously, $T=B$. For instance, $*(\sigma(b a(b))) \Rightarrow \sigma(*(b)) \Rightarrow \sigma(b)$, since both $b$ and $a(b)$ belong to $U$. But no rule is applicable to $*(\sigma(b a(a)))$. Note that $D_{1}(x)$ could as well be $T_{\Sigma}$ since $T$ can check later that the left subtree is of the required form. The essential use of the regular look-ahead is in the restriction of the right subtree to $U$.

An even more simple example was exhibited in [16]. Let $a$ be of rank 0 and $b$ of rank 2 . Then the tree transformation $\{\langle b(a a), a\rangle\}$ is not a $t-f s t$, but it is a $t^{r}-f s t$ and also a $b-f s t$.

We immediately obtain the following corollary. 
COROLLARY 2.3. $T-F S T \subset T^{R}-F S T$.

Proof. Inclusion is trivial: each $t$-fst is changed into a $t^{r}$-fst by simply specifying all variables to range over the recognizable tree language $T_{\Sigma}$, where $\Sigma$ is the input alphabet. Proper inclusion was shown in Example 2.2.

We now obtain the following two facts.

\section{COROLLARY 2.4.}

(1) The classes of tree transformations B-FST and $T^{R}-F S T$ are incomparable.

(2) $T^{R}-F S T$ is not closed under composition.

Proof. For (1), it should be clear that the $b$-fst of [BT, Example 2.1] is not a $t^{r}$ $f s t$, while Corollary 2.3 and [BT, Example 2.2] imply that there is a $t^{r}$-fst that is not a $b$-fst. For (2), note that [BT, Example 2.1] is a composition of two $t^{r}$-fst.

We now define linearity and determinism.

Definition 2.5. Let $T=\left\langle\Sigma, \Delta, Q, Q_{d}, R\right\rangle$ be a $t^{r}-f s t . T$ is linear if all righthand sides of rules in $R$ are linear. $T$ is deterministic if the following holds:

(1) $Q_{d}$ is a singleton;

(2) if $\left\langle s \rightarrow t_{1}, D_{1}\right\rangle$ and $\left\langle s \rightarrow t_{2}, D_{2}\right\rangle$ are different rules in $R$ (with the same lefthand side), then $D_{1}\left(x_{i}\right) \cap D_{2}\left(x_{i}\right)=\varnothing$ for some $i \in\{1,2, \ldots, k\}$, where $k$ is the number of variables in $s$ (for $k=0$ this means that different rules should have different lefthand sides).

Thus, in a deterministic $t^{r}$-fst, different rules may have the same lefthand side, but, in that case, the ranges of the variables are such that the two rules are never applicable in the same situation. Note that one can effectively determine whether a given $t^{r}$-fst is deterministic (RECOG is closed under intersection and has a solvable emptiness problem).

Linearity and determinism will be denoted as usual by $L$ and $D$ respectively. Note that, for a modifier $Z \in\{L, D, L D\}, Z T-F S T \subset Z T$-FST (the $t^{r}$-fst of Example 2.2 is linear and deterministic).

Since for linear $b$-fst and linear $t^{r}$-fst all properties $(B 1),(B 2)$ and $(T)$ are now "eliminated", one would expect that $L B-F S T=L T^{R}-F S T$. Before proving this we show how to decompose the $t^{r}-f s t$ : the regular look-ahead can be computed in advance by a deterministic bottom-up finite state relabeling.

THEOREM 2.6. $T^{R}-F S T \subseteq D B Q R E L \circ T-F S T$, and, for $Z \in\{L, D, L D\}$, $Z T^{R}-F S T \subseteq D B Q R E L \circ Z T-F S T$.

Proof. Let $T=\left\langle\Sigma, \Delta, Q, Q_{d}, R\right\rangle$ be a $t^{r}$-fst. Consider all "recognizable properties" which $T$ checks with its regular look-ahead. A finite state relabeling can be used to check, for a given input tree $t$, whether the subtrees of $t$ have these properties or not, and to put this information at their father nodes. After this, an ordinary $t-f s t$ can be used to simulate $T$. Formally we proceed as follows.

Let $L_{1}, \ldots, L_{n}$ be all the recognizable tree languages occurring as ranges of variables in the rules of $T$. Let $U$ denote the set $\{0,1\}^{n}$,i.e. the set of all sequences of 0 's and 1's of length $n$. For $u \in U$, the $j$ th element $(1 \leq j \leq n)$ of $u$ will be denoted by $u(j)$. Intuitively, an element $u$ of $U$ will be used to indicate membership of a tree in $L_{1}, \ldots, L_{n}\left(u(j)=1\right.$ iff the tree belongs to $\left.L_{j}\right)$. Let $\Omega$ be the ranked alphabet such 
that $\Omega_{0}=\Sigma_{0}$ and, for $k \geq 1, \Omega_{k}=\Sigma_{k} \times U^{k}$. Thus an element of $\Omega_{k}$ is of the form $\left\langle\sigma,\left\langle u_{1}, \ldots, u_{k}\right\rangle\right\rangle$ with $\sigma \in \Sigma_{k}$ and $u_{1}, \ldots, u_{k} \in U$. Intuitively, if a node is labeled by $\left\langle\sigma,\left\langle u_{1}, \ldots, u_{k}\right\rangle\right\rangle$, it means that $u_{i}$ contains all the information about the $i$ th subtree of the node. The mapping $B: T_{\Sigma} \rightarrow T_{\Omega}$ is now defined recursively as follows:

(1) for $\sigma \in \Sigma_{0}, B(\sigma)=\sigma$;

(2) for $k \geq 1, \sigma \in \Sigma_{k}$ and $t_{1}, \ldots, t_{k} \in T_{\Sigma}, B\left(\sigma\left(t_{1} \ldots t_{k}\right)\right)=\tau\left(B\left(t_{1}\right) \ldots B\left(t_{k}\right)\right)$, where $\tau=\left\langle\sigma,\left\langle u_{1}, \ldots, u_{k}\right\rangle\right\rangle$ and, for $1 \leq i \leq k$ and $1 \leq j \leq n, u_{i}(j)=1$ iff $t_{i} \in L_{i}$.

It is left to the reader to show that $B$ can be realized by a (total) deterministic bottom-up finite state relabeling (given the deterministic bottom-up finite tree automata recognizing $\left.L_{1}, \ldots, L_{n}\right)$. Next we define the top-down $f_{s t} T^{\prime}=\langle\Omega, \Delta$, $Q, Q_{d}, R^{\prime}>$ such that

(1) if $q(\sigma) \rightarrow t$ is in $R$, then it is in $R^{\prime}$;

(2) if $\left\langle q\left(\sigma\left(x_{1} \ldots x_{k}\right)\right) \rightarrow t, D\right\rangle$ is in $R$, then each rule of the form $q(\langle\sigma, \vec{u}\rangle$ $\left.\left(x_{1} \ldots x_{k}\right)\right) \rightarrow t$ is in $R^{\prime}$, where $\bar{u}=\left\langle u_{1}, \ldots, u_{k}\right\rangle \in U^{k}$ and, for $1 \leq i \leq k$ and $1 \leq j \leq n$, if $D\left(x_{i}\right)=L_{j}$ then $u_{i}(j)=1$.

This completes the construction. It should be clear that $T=B=T^{\prime}$, and that, if $T$ is linear, then so is $T^{\prime}$. It should also be obvious that, in the above construction, $U$ may be replaced by the smaller set $\left\{u \in U \mid\right.$ for all $j_{1}$ and $j_{2}$, if $L_{j_{1}} \cap L_{j_{2}}=\varnothing$, then $u\left(j_{1}\right)$ and $u\left(j_{2}\right)$ are not both 1 ) (other elements of $U$ do not occur in trees $B(t)$ ). After this replacement (which influences $T^{\prime}$ ) one can easily see that if $T$ is deterministic, then so is $T^{\prime}$.

An immediate consequence of this theorem and previous decomposition results (in $[B T]$ ) is that each element of $T^{R}-F S T$ is decomposable into elements of REL, FTA and HOM.

COROLLARY 2.7. The domain of a $t^{r}$-fst is recognizable.

Proof. Lemma 1.2(2).

We now show that the classes of linear $b$ - $f s t$ and linear $t^{r}$-fst coincide (cf. [BT, Theorem 2.8]).

THEOREM 2.8. $L T^{R_{-} F S T}=L B-F S T$.

Proof. First

$$
\begin{aligned}
L T^{R}-F S T & \subseteq D B Q R E L \circ L T-F S T \\
& \subseteq D B Q R E L \circ L B-F S T \\
& \subseteq L B-F S T
\end{aligned}
$$

by Theorem 2.6

by $[B T$, Theorem 2.8$]$

by $[B T$, Theorem $4.5(2)]$

(note that finite state relabelings are linear).

Secondly, we show that $L B-F S T \subseteq L T^{R}-F S T$. The construction is the same as that in the proof of $[B T$, Theorem 2.9], but now we can use look-ahead to handle deletion. Let $B=\left\langle\Sigma, \Delta, Q, Q_{d}, R\right\rangle$ be an arbitrary linear $b$-fst. Let, for each $q$ in $Q$, $B(q)$ denote the $b$-fst $\langle\Sigma, \Delta, Q,\{q\}, R\rangle$. Then we construct the linear $t^{r}$-fst $T=\langle\Sigma$, $\left.\Delta, Q, Q_{d}, R_{T}\right\rangle$, where $R_{T}$ is defined by the following two requirements.

(1) If $\sigma \rightarrow q(t)$ is in $R$, then $q(\sigma) \rightarrow t$ is in $R_{T}$.

(2) If $\sigma\left(q_{1}\left(x_{1}\right) \ldots q_{k}\left(x_{k}\right)\right) \rightarrow q(t)$ is in $R$, then

the rule $\left\langle q\left(\sigma\left(x_{1} \ldots x_{k}\right)\right) \rightarrow t\left[q_{1}\left(x_{1}\right), \ldots, q_{k}\left(x_{k}\right)\right], D\right\rangle$, is in $R_{T}$, where, for $1 \leq i \leq k, D\left(x_{i}\right)=\operatorname{dom}\left(B\left(q_{i}\right)\right)$. Note that $\operatorname{dom}\left(B\left(q_{i}\right)\right)$ is recognizable by $[B T$, 
Corollary 3.12]. Note also that it would suffice to have $D\left(x_{i}\right)=\operatorname{dom}\left(B\left(q_{i}\right)\right)$ for those $x_{i}$ that do not occur in $t$, and $D\left(x_{i}\right)=T_{\Sigma}$ for the other $x_{i}$.

A formal proof that $T=B$ is left to the reader. Intuitively $T$ simulates $B$ in the top-down direction by translating each node in the same piece of tree as $B$. Whenever $B$ deletes a subtree $t$ after arriving at its top in state $q$, T checks whether $t \in \operatorname{dom}(B(q))$ before deleting $t\left(t \in \operatorname{dom}(B(q))\right.$ means that there exists $s \in T_{\Delta}$ such that $t \underset{\vec{B}}{\vec{*}} q(s))$.

In the rest of this section we discuss composition of $t^{r}$-fst. We shall show (cf. property $(B 1)$ ) that, if either $T_{1}$ is deterministic or $T_{2}$ is linear, then $T_{1} \circ T_{2}$ is in $T^{R}$ $F S T$. Moreover, $D T^{R}-F S T$ and $L T^{R}-F S T$ are closed under composition. To prove these results we first consider some simple cases in the following two lemmas (concerning homomorphisms and finite state relabelings respectively).

\section{LEMMA 2.9.}

$$
\begin{aligned}
T^{R}-F S T \circ L H O M & \subseteq T^{R}-F S T, \\
D T^{R}-F S T \circ H O M & \subseteq D T^{R}-F S T .
\end{aligned}
$$

Proof. Let $T=\left\langle\Sigma, \Delta, Q, Q_{d}, R\right\rangle$ be a $t^{r}$-fst and let $H$ be a homomorphism from $T_{\Delta}$ into $T_{\Omega}$. For both cases, (1) and (2), the construction of a $t^{r}-f s t T^{\prime}$ defining $T \subset H$ is similar to that in [BT, Lemma 4.1]; look-ahead is used to handle deletion by $H$. Let $H$ be extended to $T_{\Delta}[Q(X)]$ by definining, informally, $H(q(x))=q(x)$ for all $q(x) \in Q(X)$. Let, for $p \in Q, T(p)$ denote the $t^{r}-f s t\langle\Sigma, \Delta, Q,\{p\}, R\rangle$. Note that, by Corollary 2.7, dom $(T(p)) \in R E C O G$. We now construct the $t^{r}-f s t T^{\prime}=\left\langle\Sigma, \Omega, Q, Q_{d}\right.$, $R^{\prime}>$ such that

(1) if $q(\sigma) \rightarrow t$ is in $R$, then $q(\sigma) \rightarrow H(t)$ is in $R^{\prime}$;

(2) if $\left\langle q\left(\sigma\left(x_{1} \ldots x_{k}\right)\right) \rightarrow t, D\right\rangle$ is in $R$,

then $\left\langle q\left(\sigma\left(x_{1} \ldots x_{k}\right)\right) \rightarrow H(t), D^{\prime}\right\rangle$ is in $R^{\prime}$, where, for $1 \leq i \leq k, D^{\prime}\left(x_{i}\right)$ is the intersection of $D\left(x_{i}\right)$ and all tree languages $\operatorname{dom}(T(p))$ such that $p\left(x_{i}\right)$ oecurs in $t$ but not in $H(t)$.

It is left to the reader to prove that $T \cdot H \subseteq T^{\prime}$ and that, if $H$ is linear or $T$ is deterministic, then $T^{\prime} \subseteq T_{\circ} H$ also. Note that, if $T$ is deterministic, then so is $T^{\prime}$ (the $D^{\prime}\left(x_{i}\right)$ are included in the $D\left(x_{i}\right)$ ). This proves the lemma.

\section{LEMMA 2.10.}

$$
\begin{aligned}
T^{R}-F S T \circ \quad Q R E L & \subseteq T^{R}-F S T, \\
D T^{R_{-}} F S T_{\circ} D T Q R E L & \subseteq D T^{R_{-}}-F S T, \\
D T^{R_{-}} F S T \circ D B Q R E L & \subseteq D T^{R_{-}}-F S T .
\end{aligned}
$$

Proof. We first prove (1) and (2). The proof is similar to that of $[B T$, Lemma 4.2]. Let $T=\left\langle\Sigma, \Delta, Q, Q_{d}, R_{T}\right\rangle$ be a $t^{r}$-fst and $L=\left\langle\Delta, \Omega, P, P_{d}, R_{L}\right\rangle$ a top-down finite state relabeling. We extend the input alphabet of $L$ to $\Delta \cup X$ by adding $X$ to $\Delta_{0}$. We now define a $t^{r}$-fst $K$ such that $K=T L$. Let $K=\langle\Sigma, \Omega, Q \times P$, $\left.Q_{d} \times P_{d}, R_{K}\right\rangle$, where $R_{K}$ is obtained by the following two requirements. 

$R_{K}$.

(i) If the rule $q(\sigma) \rightarrow t_{1}$ is in $R_{T}$ and $p\left(t_{1}\right) \underset{L}{\vec{L}} t_{2}$, then the rule $\langle q, p\rangle(\sigma) \rightarrow t_{2}$ is in

(ii) Let $\left\langle q\left(\sigma\left(x_{1} \ldots x_{k}\right)\right) \rightarrow t, D\right\rangle$ be in $R_{T}$. Obviously $t$ can be written as $t=s_{1}\left[q_{1}\left(x_{i_{1}}\right), \ldots, q_{m}\left(x_{i_{m}}\right)\right]$, where $s_{1} \in T_{\Delta}\left[X_{m}\right]$ is linear and nondeleting with respect to $X_{m \cdot}$ If $p\left(s_{1}\right) \stackrel{*}{\vec{L}} s_{2}\left[p_{1}\left(x_{1}\right), \ldots, p_{m}\left(x_{m}\right)\right]$, then the rule is in $R_{K}$ with the same $D$.

$\langle q, p\rangle\left(\sigma\left(x_{1} \ldots x_{k}\right)\right) \rightarrow s_{2}\left[\left\langle q_{1}, p_{1}\right\rangle\left(x_{i_{1}}\right), \ldots,\left\langle q_{m}, p_{m}\right\rangle\left(x_{i_{m}}\right)\right]$

Clearly, if $T$ and $L$ are deterministic, then so is $K$.

We now prove (3), which is the essential composition result. Let $T$ be in $D T^{R}$ $F S T$ and $B$ in $D B Q R E L$. We shall construct a transducer $T^{\prime}$ in $D T^{R}-F S T$ such that $T^{\prime}=T=B$.

Intuitively, when $T^{\prime}$ arrives at a node of the input tree, it first computes the piece of output $t$ that $T$ would produce at this node, and then runs $B$ on $t$. However, to be able to run $B$ on $t, T^{\prime}$ should know the states in which $B$ arrives at this piece of output. But, these states can be computed by regular look-ahead. The formal construction is as follows. Let $T=\left\langle\Sigma, \Delta, Q, Q_{d}, R\right\rangle$ with $Q_{d}=\left\{q_{d}\right\}$ and let $B=\left\langle\Delta, \Omega, Q_{B}, Q_{B d}, R_{B}\right\rangle$. Let as usual, for $q \in Q, T(q)$ denote $\langle\Sigma, \Delta, Q,\{q\}, R\rangle$ and, for $q \in Q_{B}, B(q)$ denote $\left\langle\Delta, \Omega, Q_{B},\{q\}, R_{B}\right\rangle$. We now construct the $t^{r}-f s t T^{\prime}=\langle\Sigma, \Omega$, $\left.Q, Q_{d}, R^{\prime}\right\rangle$, where $R^{\prime}$ is determined as follows.

(i) Let $q(\sigma) \rightarrow t$ be in $R$, where $q \in Q, \sigma \in \Sigma_{0}$ and $t \in T_{\Delta}$. Suppose that $t \vec{B} \vec{B} p\left(t^{\prime}\right)$ for some $t^{\prime} \in T_{\Omega}$ and some $p \in Q_{B}$ such that, if $q=q_{d}$ then $p \in Q_{B d^{-}}$. Then the rule $q(\sigma)$ $\rightarrow t^{\prime}$ is in $R^{\prime}$.

(ii) Let $\left\langle q_{0}\left(\sigma\left(x_{1} \ldots x_{k}\right)\right) \rightarrow t, D\right\rangle$ be in $R$, where $k \geq 1, \sigma \in \Sigma_{k}, t \in T_{\Delta}\left[Q\left(X_{k}\right)\right]$, $q_{0} \in Q$ and, for $1 \leq i \leq k, D\left(x_{i}\right) \subseteq T_{\Sigma}$. Clearly $t$ can be written as $t$ $=s\left[q_{1}\left(x_{i_{1}}\right), \ldots, q_{m}\left(x_{i_{m}}\right)\right]$ for certain $m \geq 0, s \in T_{\Delta}\left[X_{m}\right], q_{1}, \ldots, q_{m} \in Q$ and $x_{i_{1}}, \ldots, x_{i_{m}} \in X_{k}$, such that $x_{1}, \ldots, x_{m}$ all occur in $s$. Let $p_{1}, \ldots, p_{m}$ be a sequence of $m$ states from $Q_{B}$ and suppose that $s\left[p_{1}\left(x_{1}\right), \ldots, p_{m}\left(x_{m}\right)\right] \stackrel{*}{\vec{B}} p_{0}\left(s^{\prime}\right)$ for some $s^{\prime} \in T_{\Omega}\left[X_{m}\right]$ and some $p_{0} \in Q_{B}$ such that, if $q_{0}=q_{d}$ then $p_{0} \in Q_{B d}(B$ is of course extended to trees with variables in the usual way). Then the rule $q_{0}\left(\sigma\left(x_{1} \ldots x_{k}\right)\right)$ $\rightarrow s^{\prime}\left[q_{1}\left(x_{i_{1}}\right), \ldots, q_{m}\left(x_{i_{m}}\right)\right]$ is in $R^{\prime}$, where the ranges of $x_{1}, \ldots, x_{k}$ are specified by $D^{\prime}$ as follows. For $1 \leq u \leq k, D^{\prime}\left(x_{u}\right)$ is the intersection of $D\left(x_{u}\right)$ and all tree languages $\operatorname{dom}\left(T\left(q_{j}\right) \circ B\left(p_{j}\right)\right)$ such that $x_{i_{j}}=x_{u}$. Note that these tree languages are recognizable by Lemma 1.2 and the fact that each $t^{r}$-f st can be decomposed into $b$ fst (Theorem 2.6).

This ends the construction of $T^{\prime}$. It is left to the reader to check that $T^{\prime}$ is deterministic (using the determinism of $T$ and $B$ ) and to prove that $T^{\prime}=T: B . \square$

We can now prove the composition results for $t^{r}-f s t$.

\section{THEOREM 2.11 .}

$$
\begin{aligned}
T^{R}-F S T \circ L T^{R}-F S T & \subseteq T^{R}-F S T, \text { and } \\
L T^{R}-F S T \circ L T^{R}-F S T & \subseteq L T^{R}-F S T . \\
D T^{R_{-}} F S T \circ T^{R}-F S T & \subseteq T^{R}-F S T, \text { and } \\
D T^{R_{-}} F S T \circ D T^{R_{-}} F S T & \subseteq D T^{R_{-}}-F S T .
\end{aligned}
$$

Proof.

(1) The second inclusion is immediate by Theorem 2.8 and [BT, Theorem 4.5(2)]. The first inclusion can be shown as follows. 


$$
\begin{aligned}
T^{R}-F S T \circ L T^{R}-F S T \\
\quad=T^{R}-F S T \cdot L B-F S T \\
\quad \subseteq T^{R}-F S T \cdot Q R E L=L H O M \\
\quad \subseteq T^{R}-F S T \circ L H O M \\
\quad \subseteq T^{R}-F S T
\end{aligned}
$$

by Theorem 2.8

by $[B T$, Theorem $3.15(2)]$

by Lemma $2.10(1)$

by Lemma $2.9(1)$.

(2) For both inclusions we have that

$$
\begin{aligned}
& D T^{R}-F S T \circ(D) T^{R}-F S T \\
& \subseteq D T^{R}-F S T \circ D B Q R E L \circ(D) T-F S T \\
& \subseteq D T^{R}-F S T \circ(D) T-F S T \\
& \subseteq D T^{R}-F S T \circ H O M \circ L(D) T-F S T \\
& \subseteq D T^{R}-F S T \circ L(D) T-F S T
\end{aligned}
$$
by Theorem 2.6
by Lemma $2.10(3)$
by Lemma $1.1(1)$
by Lemma $2.9(2)$.

Now DT $T^{R}-F S T \circ L T-F S T \subseteq T^{R}-F S T$ by (1) of this theorem, and

$$
\begin{aligned}
& D T^{R}-F S T \cdot L D T-F S T \\
& \subseteq D T^{R}-F S T \circ D T Q R E L \circ L H O M \\
& \subseteq D T^{R}-F S T \circ L H O M \\
& \subseteq D T^{R}-F S T
\end{aligned}
$$

by Lemma 1.1(2)

by Lemma $2.10(2)$

by Lemma $2.9(2)$.

This proves the theorem.

It is left to the reader to show that $L D T^{R}-F S T$ is closed under composition.

Note that it follows from Theorem 2.11 that the inclusion signs in Theorem 2.6 may be replaced by equality signs. Thus $T^{R}-F S T=D B Q R E L \circ T-F S T$. We finally mention a result similar to $[B T$, Theorem 3.7$]$ (see also $[B T$, Theorem $5.15])$.

THEOREM 2.12. $T^{R}-F S T=H O M \circ L T^{R}-F S T$.

Proof. The inclusion $H O M \circ L T^{R}-F S T \subseteq T^{R}-F S T$ is immediate from Theorem 2.11. The inclusion $T^{R}-F S T \subseteq H O M \circ L T^{R}-F S T$ can be shown in much the same way as in the proof of T-FST $\subseteq H O M \circ L T-F S T$ [BT, Lemma 3.6]. The only additional problem is the regular look-ahead: the image of a recognizable tree language under a homomorphism need not be recognizable. The solution is to consider a homomorphism $H$ from $T_{\Sigma}$ to $T_{\Sigma}$ (see the proof of [BT, Lemma 3.6] for notation) such that, for all $t$ in $T_{\Sigma}, H\left(T_{1}(t)\right)=t$. The easy definition of $H$ is left to the reader. Now, if in a rule of the $t^{r}-f s t T$, the recognizable tree language $U$ occurs as look-ahead, then we can use $H^{-1}(U)$ as look-ahead in the corresponding rule of $T_{2}$. Note that $H^{-1}(U) \in R E C O G$ (cf. Lemma 1.2(1)). The details of the proof are left to the reader.

COROLLARY 2.13. $T^{R}-F S T=T-F S T \circ L H O M$.

Proof. By Theorems 2.12 and $2.8, T^{R}-F S T=H O M \circ L B-F S T$. From the proof of (7) in [BT, section 6] it follows that HOM LB-FST $=T-F S T \circ L H O M$.

\section{Comparison of deterministic fst}

The classes of tree transformations DB-FST and DT-FST are incomparable. In fact there are several reasons for the incomparability of these classes. We now 
consider some typical $d b$-fst and $d t$-fst capabilities respectively. We start by considering advantages of DB-FST over DT-FST.

Firstly we note that property $(B 1)$ is eliminated, but property $(B 2)$ is not. Thus $D B-F S T$ contains elements not even in T-FST (obviously, the $b-f s t B$ in $[B T$, Example 2.6] is in DB-FST).

Secondly, a $d b-f s t$ can recognize the "lowest" occurrence of some symbol in a tree (since it is the first occurrence), but this cannot be done by a $d t$-fst (since it is the last occurrence for him).

Thirdly, it is well known (see for instance [15]) that there are recognizable tree languages which cannot be recognized by a deterministic top-down finite tree automaton. The next theorem shows that such languages cannot be the domain of any deterministic $t$-fst (cf. [11]).

THEOREM 3.1. A tree language is the domain of a deterministic $t$-fst if and only if it is the domain of a deterministic top-down fta.

Proof. The if-direction is trivial. To prove the only-if direction, let $T=\langle\Sigma, \Delta$, $Q, Q_{d}, R>$ be a $d t-f s t$. We may assume that for all $k \geq 1, \sigma \in \Sigma_{k}$ and $q \in Q$ there is a rule with lefthand side $q\left(\sigma\left(x_{1} \ldots x_{k}\right)\right)$ in $R$. We construct the deterministic topdown $f t a F=\left\langle\Sigma, \Sigma, Q^{\prime}, Q_{\mathrm{d}}^{\prime}, R^{\prime}\right\rangle$ such that $Q^{\prime}$ is the powerset of $Q, Q_{\mathrm{d}}^{\prime}=\left\{Q_{\mathrm{a}}\right\}$ and $R^{\prime}$ is defined as follows.

(1) For $k \geq 1, A \subseteq Q$ and $\sigma \in \Sigma_{k}$, the rule $A\left(\sigma\left(x_{1} \ldots x_{k}\right)\right) \rightarrow \sigma\left(A_{1}\left(x_{1}\right) \ldots A_{n}\left(x_{n}\right)\right)$ is in $R^{\prime}$, where $A_{i}=\left\{p \in Q \mid\right.$ there is a rule $q\left(\sigma\left(x_{1} \ldots x_{k}\right)\right) \rightarrow t$ in $R$ such that $q \in A$ and $p\left(x_{i}\right)$ occurs in $\left.t\right\}$.

(2) For $A \subseteq Q$ and $\sigma \in \Sigma_{0}, A(\sigma) \rightarrow \sigma$ is in $R^{\prime}$ if and only if for all $q \in A$ there is a rule with lefthand side $q(\sigma)$ in $R$ (note that in particular $\varnothing(\sigma) \rightarrow \sigma$ is in $R^{\prime}$ ).

It is left to the reader to show that $\operatorname{dom}(F)=\operatorname{dom}(T)$. Intuitively, the state of $F$ at some node contains all states in which $T$ arrives at copies of this node (made by $T$ when processing higher nodes). At the leaves, $F$ checks whether all these states are final states of $T$.

Next we consider advantages of DT-FST over DB-FST. First we note that property $(T)$ is not eliminated : a $d t-f s t$ has the ability to copy an input subtree and to continue translation of these copies in different states. Thus the $d t-f s t$ which translates every tree $\sigma(b(b(. \ldots b(a) \ldots)))$ into $\tau\left(b\left(\left(\ldots b\left(a_{1}\right) \ldots\right)\right) b\left(b\left(\ldots b\left(a_{2}\right) \ldots\right)\right)\right)$ is not in $B-F S T$.

Secondly, a $d t$-fst can recognize the "highest" occurrence of some symbol in a tree, but this cannot be done by a $d b-f s t$.

Thirdly, a $d t-f s t$ can distinguish between left and right, but a $d b-f s t$ is not able to see this difference, because it starts at the bottom.

This concludes our comparison of DB-FST and DT-FST. The reader might have noticed that the mentioned advantages of $D B-F S T$ over DT-FSTcan all be handled by the use of regular look-ahead. Also, those of DT-FST over DB-FST can be eliminated by restricting the number of states of the $d t-f s t$ to one. We now show that this holds in general. Let $O D T^{R}-F S T$ denote the class of $d t^{r}$-fst $\langle\Sigma, \Delta, Q$, $Q_{d}, R>$ such that $Q=Q_{d}$, i.e. the class of one-state deterministic $t^{r}$-fst.

THEOREM 3.2. $O D T^{R_{-} F S T}=D B-F S T \subset D T^{R_{-F}} F S$.

Proof. Inclusion of DB-FST in $D T^{R}-F S T$ is proved as follows. 
$D B-F S T$

$$
\begin{aligned}
& \subseteq D B Q R E L \circ H O M \\
& \subseteq D T^{R}-F S T \circ D B Q R E L \circ H O M \\
& \subseteq D T^{R}-F S T \circ H O M \\
& \subseteq D T^{R}-F S T
\end{aligned}
$$

by $[B T$, Theorem $3.15(3)]$

(since the identity is in $D T^{R}-F S T$ )

by Lemma $2.10(3)$

by Lemma $2.9(2)$.

Since the identity can be realized by a one-state $d t^{r}$-fst and since the constructions in Lemmas 2.9 and 2.10(3) preserve the number of states, DB-FST is included in $O D T^{R}-F S T$. The properness of the inclusion of $D B-F S T$ in $D T^{R}-F S T$ follows from the discussion preceding this theorem. Inclusion of $O D T^{R}-F S T$ in $D B-F S T$ can be proved as follows. By Theorem 2.6, DT $T^{R}-F S T \subseteq D B Q R E L \circ D T$ FST. Moreover, from the construction in the proof of that theorem it follows that every one-state $d t^{r}$-fst is the composition of an element of $D B Q R E L$ and a onestate $d t-f s t$. It is left to the reader to show that each one-state $d t-f s t$ is in DB-FST. The required inclusion now follows from the closure of DB-FST under composition ([BT, Theorem 4.6(2)]).

Thus the addition of regular look-ahead to T-FST has made the deterministic bottom-up fst into a proper subclass of the deterministic top-down fst (with regular look-ahead).

\section{Surface sets and tree transformation languages}

In this section we show how the results of the previous sections can be used to prove properties of surface sets and tree transformation languages, in particular closure properties.

Notation 4.1. Throughout this section, $\mathscr{L}$ denotes a fixed family of tree languages closed under deterministic bottom-up finite state relabelings (i.e. elements of $D B Q R E L$ ).

Note that $D B Q R E L$ is included in both $L B-F S T$ and DB-FST. Note also that for instance $R E C O G$ is closed under DBQREL.

We first show that regular look-ahead has no influence on surface sets: the classes of $(T-F S T, \mathscr{L})$ and $\left(T^{R}-F S T, \mathscr{L}\right)$ surface sets are equal.

\section{THEOREM 4.2.}

$$
\begin{aligned}
T^{R}-F S T(\mathscr{L}) & =T-F S T(\mathscr{L}) \\
D T^{R}-F S T(\mathscr{L}) & =D T-F S T(\mathscr{L}) \\
L T^{R}-F S T(\mathscr{L}) & =\operatorname{LT}-F S T(\mathscr{L})=\operatorname{LB}-F S T(\mathscr{L})
\end{aligned}
$$

Proof. Follows immediately from the decomposition result of Theorem 2.6 (and, for (3), Theorem 2.8).

Obviously a similar result for tree transformation languages is obtained by applying yield to the above equations.

From this theorem and the composition results in Theorem 2.11 we obtain a number of closure properties of surface sets, some of which are expressed in the next theorem. 


\section{THEOREM 4.3.}

(1) $T-F S T(\mathscr{L})$ is closed under linear fst.

(2) DT-FST $(\mathscr{L})$ is closed under deterministic bottom-up and top-down fst.

Proof. Immediate from Theorem 4.2, Theorem 2.11 and Theorem 3.3.

Theorem 4.3(1) was proved by Baker [3, Theorem 1.2.5] by generalizing Rounds' proof [13] for the special case $\mathscr{L}=R E C O G$. Closure of $D T$ $F S T(R E C O G)$ under $d t-f s t$ was proved by Rounds [13].

These theorems can easily be extended to surface sets which are obtained by repeated application of top-down $f s t$. In fact, the next theorem shows that the regular look-ahead can be "taken out of" any sequence of $t^{r}$-fst. Let, for any class $\mathscr{F}$ of tree transformations, $\mathscr{F}^{k}$ be defined by $\mathscr{F}^{1}=\mathscr{F}$ and $\mathscr{F}^{k+1}=\mathscr{F}^{k} \circ \mathscr{F}$.

THEOREM 4.4. For eack $k \geq 1$, (1) $\left(T^{R}-F S T\right)^{k}=D B Q R E L \circ(T-F S T)^{k}$, (2) $\left(T^{R}-F S T\right)^{k} \circ D T^{R}-F S T=D B Q R E L \circ(T-F S T)^{k} \circ D T-F S T$.

Proof. (1) We first show that $T^{R}-F S T \circ T^{R}-F S T=T^{R}-F S T \circ T-F S T$. One inclusion is trivial. The other inclusion is proved as follows:

$$
\begin{array}{rlrl}
T^{R}-F S T \circ T^{R}-F S T & & \\
& \subseteq T^{R}-F S T \circ D B Q R E L \circ T-F S T & & \text { by Theorem } 2.6 \\
& \subseteq T^{R}-F S T \circ T-F S T & & \text { by Lemma } 2.10(1) .
\end{array}
$$

From this, and the fact that $T^{R}-F S T=D B Q R E L \circ T-F S T$ (see section 2), (1) easily follows. The proof of (2) is similar.

From this theorem it follows for instance that $(T-F S T)^{k}(\mathscr{L})=\left(T^{R}-F S T\right)^{k}(\mathscr{L})$, and hence $(T-F S T)^{k}(\mathscr{L})$ is closed under linear $f_{s t}([3$, Corollary 1.2.7]). Similarly, $D T-F S T\left((T-F S T)^{k}(\mathscr{L})\right)$ is closed under deterministic bottom-up and top-down fst.

Let us now turn to tree transformation languages. Recall that we have introduced a symbol $e$ such that yield $(e)=\lambda$. We note first that it follows from Theorem 4.3 that Lemma 1.3 holds for both yield $(T-F S T(\mathscr{L}))$ and yield $(D T$ $F S T(\mathscr{L})$ ). We express this informally in the following corollary.

COROLLARY 4.5 Both (T-FST, $\mathscr{L})$ and $(D T-F S T, \mathscr{L})$ tree transformation languages can be "generated without $\lambda$-rules" (modulo $\lambda$ ).

It should be clear that from Theorem 4.3 other closure properties for these tree transformation languages can be inferred. Since the closure properties of yield(T-FST(L)) have been discussed thoroughly by Baker [3], we restrict ourselves to the following closure property of deterministic tree transformation languages.

THEOREM 4.6. The class of tree transformation languages yield(DT$F S T(\mathscr{L}))$ is closed under deterministic gsm mappings.

Proof. Let $\Sigma$ and $\Delta$ be ranked alphabets with $e \in \Sigma_{0}$ and $e \in \Delta_{0}$. Let $S=\left\langle K, \Sigma_{0}\right.$ $\left.-\{e\}, \Delta_{0}-\{e\}, \delta, q_{0}, F\right\rangle$ be a deterministic $g s m$ (for notation, see [10, sections 9.3 and 12.3]). We shall show that there exists a deterministic top-down fst $T$ with regular look-ahead such that, for every $t \in T_{\Sigma}$, if yield $(t)$ is not accepted by $S$, then $t$ 
is not accepted by $T$, and if yield $(t)$ is accepted by $S$, then so is $t$ by $T$ and yield $(T(t))=S($ yield $(t))$. Consequently, for any tree language $L \subseteq T_{\Sigma}, \operatorname{yield}(T(L))$ $=S($ yield $(L))$. The theorem then easily follows from the closure of $D T^{R}-F S T$ under composition (Theorem 2.11(2)).

$T$ is constructed as follows (the construction being a variation on a known theme). Let, for $q_{1}, q_{2} \in K, R\left(q_{1}, q_{2}\right)$ denote the recognizable tree language consisting of all trees $t \in T_{\Sigma}$ such that $\delta\left(q_{1}\right.$, yield $\left.(t)\right)=\left\langle q_{2}, w\right\rangle$ for some output string $w \in\left(\Delta_{0}-\{e\}\right)^{*}$ (thus, when started in state $q_{1}, S$ arrives in state $q_{2}$ after processing yield $(t))$. Recognizability of $R\left(q_{1}, q_{2}\right)$ follows from a straight forward extension, to handle $e$, of [13, section 3, Lemma 2]. Let now $T=\left\langle\Sigma, \Delta, Q, Q_{d}, R\right\rangle$, where $Q=(K \times K) \cup\left\{q_{s}\right\}$ (with $q_{s}$ new), $Q_{d}=\left\{q_{s}\right\}$ and the rules of $R$ are defined as follows.

(1) For $k \geq 1, \sigma \in \Sigma_{k}$ and $q_{1}, q_{2}, \ldots, q_{k+1} \in K$, the rule $\left\langle q_{1}, q_{k+1}\right\rangle\left(\sigma\left(x_{1} \ldots x_{k}\right)\right)$ $\rightarrow \sigma\left(\left\langle q_{1}, q_{2}\right\rangle\left(x_{1}\right)\left\langle q_{2}, q_{3}\right\rangle\left(x_{2}\right) \ldots\left\langle q_{k}, q_{k+1}\right\rangle\left(x_{k}\right)\right)$ is in $R$, where the range of variable $x_{i}$ is $D\left(x_{i}\right)=R\left(q_{i}, q_{i+1}\right)$.

(2) For $\sigma \in \Sigma_{0}-\{e\}$ and $\left\langle q_{1}, q_{2}\right\rangle \in K \times K$, if $\delta\left(q_{1}, \sigma\right)=\left\langle q_{2}, w\right\rangle$ for some $w \in\left(\Delta_{0}\right.$ $-\{e\})^{*}$, then the rule $\left\langle q_{1}, q_{2}\right\rangle(\sigma) \rightarrow t$ is in $R$, where $t$ is some tree in $T_{\Delta}$ such that yield $(t)=w$ (note that, if $w=\lambda$, one can take $t=e$ ).

(3) For $q \in K$, the rule $\langle q, q\rangle(e) \rightarrow e$ is in $R$.

(4) For $k \geq 1, \sigma \in \Sigma_{k}$ and $q_{1}, \ldots, q_{k+1} \in K$, if $q_{1}=q_{0}$ and $q_{k+1} \in \mathrm{F}$, then the rule $q_{s}\left(\sigma\left(x_{1} \ldots x_{k}\right)\right) \rightarrow \sigma\left(\left\langle q_{1}, q_{2}\right\rangle\left(x_{1}\right) \ldots\left\langle q_{k}, q_{k+1}\right\rangle\left(x_{k}\right)\right)$ is in $R$, where the range of $x_{i}$ is $R\left(q_{i}, q_{i+1}\right)$.

(5) For $\sigma \in \Sigma_{0}-\{e\}$, if $\delta\left(q_{0}, \sigma\right)=\left\langle q_{f}, w\right\rangle$ for some $q_{f} \in F$ and $w \in\left(\Delta_{0}-\{e\}\right)^{*}$, then the rule $q_{s}(\sigma) \rightarrow t$ is in $R$, where $t$ is a tree such that yield $(t)=w$.

(6) If $q_{0} \in F$, then $q_{s}(e) \rightarrow e$ is in $R$.

This ends the construction of $T$. It should be clear that $T$ is deterministic and that $T$ satisfies the requirements.

Note that it follows from this theorem that yield(DT-FST(L)) is closed under string homomorphisms and intersection with a regular language.

We finally mention that these results can directly be applied to certain classes of Lindenmayer languages (see also [1]).

Let $M O N$ be the class of monadic recognizable tree languages (a tree language is monadic if all symbols appearing in its trees are of rank 0 or 1 ; in [8] the number of symbols of rank 0 is restricted to one, but this is not essential for what follows).

It was shown in $[1,5,8]$ that $E T O L=\operatorname{yield}(T-F S T(M O N))$ and $E D T O L$ = yield(DT-FST $(M O N)$ ), where ETOL and EDTOL are classes of Lindenmayer languages defined in for instance [9]. Thus, since $M O N$ is obviously closed under $D B Q R E L$, Corollary 4.5 implies the well known fact that (modulo $\lambda$ ) ETOL and $E D T O L$ languages can be generated without $\lambda$-rules. From Theorem 4.6 we directly obtain the following useful result (cf. [6]).

COROLLARY 4.7. EDTOL is closed under deterministic gsm mappings.

For any $\mathscr{L} \subseteq M O N$ (with certain closure properties) yield(T-FST(L)) and yield $(D T-F S T(\mathscr{L}))$ are equal to the $\mathscr{L}$-controlled $E T O L$ languages and the $\mathscr{L}$ controlled EDTOL languages respectively (see [2]; for $L \in \mathscr{L}$, only those sequences of tables which are in $L$ may be used in the generation of the ETOL 
language). It follows that the above results are also applicable to controlled ETOL and EDTOL languages.

REFERENCES

[1] A. ARnold and M. Dauchet, Transductions de forets regulieres monadiques; forets coregulieres, RAIRO 10 (1976), 5-28.

[2] P. R. J. AsvelD, Controlled iteration grammars and full hyper-AFL's, Memoradum 114, Technical University Twente, Holland, 1976.

[3] B. S. BAKER, Tree transductions and families of tree languages, Ph.D. Thesis, Harvard University, Report TR-9-73, 1973 (also: 5th Theory of Computing, 200-206).

[4] K. ČLLIK II and R. COHEN, LR-regular grammars-an extension of LR(k) grammars, JCSS 7 (1973), 66-96.

[5] P. J. Downey, Tree transducers and ETOL tree systems (abstract), Conference on Formal Languages, Automata and Development, Noordwijkerhout, Holland, 1975.

[6] A. EhreNFEuChT and G. ROZENBERG, On inverse homomorphic images of deterministic ETOL languages, $\operatorname{LOCOS} 13$, Utrecht University, Holland, 1974.

[7] J. ENGELFRIET, Bottom-up and top-down tree transformations-a comparison, Math. Syst. Theory 9 (1975), 198-231. This paper is also referred to as BT.

[8] J. Engelfriet, Surface tree languages and parallel derivation trees, DAIMI Report PB-44, Aarhus University, Denmark, 1975 (to appear in Theoretical Computer Science).

[9] G. T. Herman and G. RozenBerg, Developmental systems and languages, North-Holland Publ. Co., Amsterdam, 1975.

[10] J. E. HoPCROFT and J. D. UlLman, Formal languages and their relation to automata, AddisonWesley Publ. Co., Reading, Mass., 1969.

[11] L. S. LeVy and A. K. Joshi, Some results in tree automata, Math. Syst. Theory 6 (1973), 334-342.

[12] W. F. OGDen and W. C. Rounds, Composition of $n$ transducers, 4th Symp. on Theory of Computing, 1972, pp. 198-206.

[13] W. C. Rounds, Mappings and grammars on trees, Math. Syst. Theory 4 ((1970), 257-287.

[14] J. W. Thatcher, Generalized ${ }^{2}$ sequential machine maps, JCSS 4 (1970), 339-367.

[15] J. W. Thatcher, Tree automata: an informal survey, in: Currents in the Theory of Computing (ed. A. V. Aho), Prentice-Hall, 1973, pp. 143-172.

[16] M. DaucheT, Transductions inversibles de forets, These, Univ. de Lille, France, 1975.

Received September 18, 1975 and in revised form December 2, 1976. 